\title{
METODOLOGÍA PARA MINIMIZAR LOS TIEMPOS DE LLEGADA EN UNA RED DE RIEGO A PRESIÓN
}

\author{
Jiménez Bello, MA¹. (P); Manzano-Juárez², J, Alonso Campos, JC ${ }^{3}$, Martínez Alzamora F. ${ }^{4}$
}

${ }^{1}$ Profesor Contratado Doctor de la Universitat Politècnica de València, Inst. U. Ingeniería del Agua y M.A (IIAMA), Camino de Vera, s/n 46022 Valencia. mijibar@dihma.upv.es

${ }^{2}$ Profesor Contratado Doctor de la Universitat Politècnica de València, Centro Valenciano de Estudios sobre el Riego, Camino de Vera, s/n 46022 Valencia. juamanju@agf.upv.es

${ }^{3}$ Ing. Industrial, , Inst. U. Ingeniería del Agua y M.A (IIAMA), Universitat Politècnica de València, Camino Vera, bq 5J, bajo 46022 Valencia, joaalcam@etsii.upv.es

${ }^{4}$ Catedrático de la Universitat Politècnica de València, Inst. U. Ingeniería del Agua y M.A (IIAMA), del Agua y del Medio Ambiente, Camino de Vera, s/n 46022 Valencia. fmartine@hma.upv.es

\section{Resumen}

Las redes de riego a presión automatizadas permiten llevar a cabo la fertirrigación centralizada y realizar operaciones de mantenimiento como la limpieza de subunidades y la eliminación de especies invasoras como el mejillón cebra.

Para limpiar la red de una determinada sustancia o hacerla llegar a todos los puntos de consumo en el menor tiempo posible y con la mínima cantidad de volumen empleado, se han diseñado dos metodologías que utilizan el modelo hidráulico de la red y algoritmos genéticos multiobjetivo paralelo. La primera tiene en cuenta la variabilidad con el tiempo de la presión en cabecera y la segunda asume que ésta es constante o no baja de un determinado valor. Con la segunda se consigue minimizar los tiempos de llegada a los puntos de consumo y reducir el volumen inyectado hasta el mínimo de reemplazo, es decir, el volumen de las tuberías de la red.

\section{1- Introducción}

Las redes colectivas de riego a presión permiten el uso de la fertirrigación colectiva, de tal forma que se puedan cubrir los requerimientos hídricos y de nutrientes utilizando un mismo sistema de distribución. Esta práctica, ha sido incorporada en numerosas comunidades de regantes con monocultivo y explotaciones de tamaño pequeño como las del este mediterráneo español.

A nivel de red de transporte, la distribución del fertilizante ha sido analizada, desde la perspectiva de la gestión, en el trabajo realizado por Jimenez-Bello et al. (2011). En él, se analiza cómo se distribuye el fertilizante en la red y cómo la programación del riego influye en el tiempo efectivo de fertilización (EFT). Se propuso una metodología para homogeneizar la EFT en el caso de que existan riegos sin fertilización y la presencia de tomas que no quieren recibir fertilizantes. Del mismo modo, en el caso de que el suministro tenga dependencia energética, la organización del riego más eficiente desde el punto de vista energético no tiene por qué coincidir con la distribución que garantiza una mejor EFT para cada una de las tomas. Por ello se propuso una metodología que combine aquellas agrupaciones de tomas óptimas 
desde el punto de vista energético con aquellas que permiten aumentar la EFT. Las conclusiones que se obtuvieron fueron que la existencia de tomas que no desean realizar fertilización disminuyen la uniformidad de EFT y difícilmente puede garantizarse que no llegue ninguna cantidad de fertilizante a las mismas.

Sin embargo, la introducción de nuevos cultivos en los distritos de riego plantea nuevos retos a la hora de realizar una gestión eficiente, puesto que no todos tienen las mismas necesidades de fertilizantes, ni se les aplican los mismos tratamientos. Otro reto importante a abordar es la posible presencia de cultivos ecológicos en la red. Ello implica limpiar la red lo más rápido posible de las sustancias existentes para adecuarlas a las necesidades de los cultivos que se riegan en un determinado momento.

Del mismo modo, la realización de operaciones de mantenimiento, como la limpieza de subunidades, conlleva inyectar ácido nítrico, fosfórico, sulfúrico o clorhídrico, cuyo pH debe ser de 2 a 3 para conseguir un buen resultado (CAJAMAR, 2014). Una referencia es inyectar $6 \mathrm{I} \mathrm{m}^{-3}$ de ácido, por lo que interesa minimizar la cantidad utilizada para rebajar el coste de la limpieza.

Otra operación frecuente es el uso de desinfectantes para la eliminación del mejillón cebra (Confederación hidrográfica del Ebro, 2014). Para este fin se utilizan métodos de control físicos y químicos. Los métodos químicos se pueden utilizar de forma preventiva, cuando se produce la entrada de las larvas en las instalaciones, o reactiva, en cuyo caso la estrategia elegida dependerá del grado de colonización para determinar la dosificación del producto y el tiempo de exposición. Las sustancias que se utilizan son oxidantes como clorados y ozono que reaccionan con el medio, este último a una mayor velocidad; y los no oxidantes como el Sulfato de Aluminio, el Nitrato Amónico, el Metasulfito de Sodio, el Sulfato de Cobre y el Potasio. Estas sustancias se pueden asumir que no reaccionan con el medio.

\section{2- Objetivos}

En este trabajo se presentan dos propuestas que permiten minimizar el tiempo de lavado de la red, o lo que es lo mismo, hacer que una sustancia llegue lo más rápido posible a todos los puntos de consumo para permitir la aplicación de distintos tratamientos, a la vez que se garantizan las presiones mínimas de funcionamiento. En caso de utilizarse una sustancia para un tratamiento, se puede además minimizar la cantidad utilizada. La primera metodología tiene en cuenta que la presión en la cabecera de la red no es constante y la segunda que sí lo es.

\section{3- Metodología}

\subsection{Caso de estudio}

El caso de estudio elegido es el sector XI de la Acequia Real del Júcar (ARJ), localizado en el término municipal de Algemesí (Valencia). Se trata de una red colectiva de 88 hidrantes multiusuarios, con 565 tomas de riego con válvulas de apertura automatizadas, 14.9 $\mathrm{km}$ de tuberías y 434 ha regadas donde el $60 \%$ son caquis, el $27 \%$ cítricos y la superficie restante se destina a otro tipo de frutales. El cabezal de riego se encuentra a una cota de $28.14 \mathrm{~m}$ y las cotas de los hidrantes varían entre $21.12 \mathrm{~m}$ y $32.6 \mathrm{~m}$. La ¡Error! No se encuentra el origen de la referencia. muestra el trazado de la red hidráulica. 


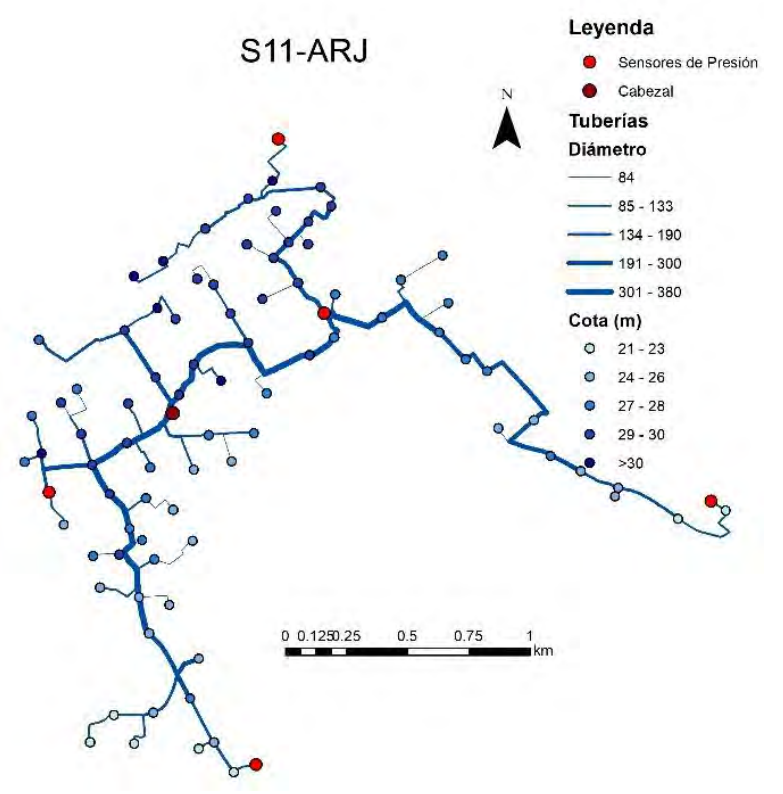

Figura 1 Topología de la red de riego del Sector XI de la Acequia Real del Júcar

El suministro se realiza a través de una red general de alta presión que abastece a otros 90 cabezales de riego. De estos, en la actualidad en 36 se ha completado el proceso de modernización a riego a presión, lo que supone un $22 \%$ de las 15.000 ha de las que consta la ARJ. El resto sigue operando mediante riego por gravedad. La regulación de la red general garantiza que la presión en cabecera no supere los $43 \mathrm{~m}$, mientras que la presión mínima no está garantizada. En el periodo de mayores requerimientos hídricos las tomas riegan tres veces al día, en periodos de 1 hora de duración. La secuencia de apertura de las tomas está repartida a lo largo del día de manera empírica para reducir las pérdidas de carga en la red de distribución y dar el servicio con las presiones mínimas requeridas en cada hidrante.

\subsection{Calibración del modelo}

Una vez confeccionado el modelo hidráulico de la red para su simulación en EPANET (Rossman, 2000), se procedió a su calibración. Para ello se colocaron 5 sensores de presión (SSC2035 Sensortechnics, Puchheim, Germany) con una frecuencia de toma de datos de 2 minutos. La instalación disponía en cabecera de un sensor que recogía los datos con una frecuencia de 1 minuto. La disposición de los sensores en los hidrantes se muestra en la ¡Error! No se encuentra el origen de la referencia..

Comparando las medidas reales con las simuladas, y tras corregir ligeramente la cota de los manómetros, el error cuadrático medio fue de $0.711 \mathrm{~m}$ y $0.709 \mathrm{~m}$ para las dos fechas que se realizó la calibración (11/8/2019 y 12/08/2019). No fue necesario modificar ningún otro parámetro inicial introducido.

La Figura 2 muestra las presiones medias, máximas, mínimas y la desviación estándar de 12 días durante la campaña de riego (1/8/2019-12/8/2019) registradas en el cabezal. Las variaciones de presión en la red principal se deben a la apertura de tomas en las diferentes redes de riego de las que se compone la ARJ y la descarga a través de ella en las acequias a presión atmosférica de las infraestructuras que continúan funcionando por gravedad. 


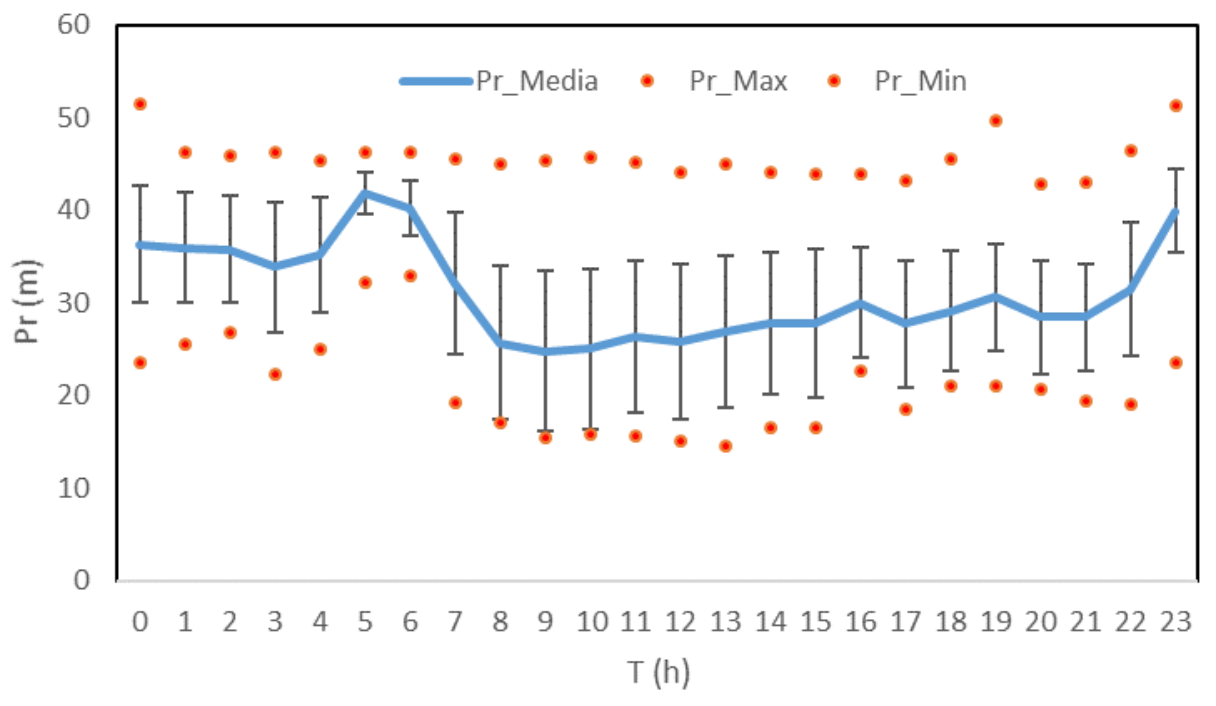

Figura 2 Presiones medias (Pr_Media), desviación estándar y presiones máximas (Pr_Max) y mínimas (Pr_Min) para el periodo del 1/8/2019 al 12/8/2019.

\subsection{Metodología multiobjetivo asumiendo que la presión en cabecera es variable (Met1)}

Para resolver el problema se ha combinado el uso de un modelo hidráulico de la red mediante la Toolkit de EPANET 2.0 (Rossman, 2000) con un algoritmo genético multiobjetivo paralelo utilizando el método NSGA-II (Deb et al., 2002).

La primera función objetivo del problema es maximizar el número de hidrantes a los que le llega la sustancia $\left(\mathrm{H}_{\mathrm{s}}\right)$.

$$
\operatorname{Max}\left(N_{H s}\right)=\sum_{i=1}^{N_{H s}}\left(T_{H_{i}}<T_{\text {JRieg }}\right)
$$

donde $T_{H i}$ es el tiempo que tarda el agua en llegar al hidrante $H_{i}$ desde el origen, $T_{J R i e g}$ es la duración de la jornada de riego, y $N_{H s}$ es el número de hidrantes del total de hidrantes de la red $N_{H}$ a los que le llega la sustancia dentro del tiempo $T_{J r i e g}$. Una vez llega la sustancia a una de las tomas de un hidrante, se considera que lo ha hecho a todas las tomas que alimenta el hidrante.

La segunda función objetivo es minimizar el déficit de presión en las tomas (DPT) o en los hidrantes (DPH) que se produce cuando se suministran los caudales requeridos:

$$
D P T=\frac{1}{N t}\left(\sum_{i=1}^{i=N t} \operatorname{Min}\left\{p_{\text {min,req }}^{t}-p_{\text {min,calc }}^{t}\right), 0\right\}
$$

donde $p_{\text {min,req }}$ es la presión mínima requerida en la toma $t, p_{\text {min,calc }}$ es la presión mínima calculada mientras esta opera y $N_{t}$ el número total de tomas que operan durante $T_{J R i e g}$

También se ha propuesto una tercera función objetivo que consiste en minimizar el volumen inyectado en la red $\left(V_{T o t}\right)$ para conseguir el primer objetivo, asumiendo que cuanto menor sea $V_{\text {Tot }}$, independientemente de la concentración del producto utilizado en la operación, normalmente en una jornada de riego $\left(T_{J R i e g}\right)$, menor será la cantidad utilizada de éste.

$$
\operatorname{Min}\left(V_{T o t}\right)=\sum_{1}^{N_{t}} V_{t}
$$


donde $V_{t}$ es el volumen consumido por cada toma $t$ y $N_{t}$ el número total de tomas que operan.

Las variables de decisión de las que se compone cada uno de las posibles soluciones (cromosomas) son los genes. Estos genes se han codificado como variables enteras en las que se determina cuándo se inicia el riego, discretizado conforme el intervalo de tiempo de la simulación hidráulica $\left(T_{\text {Hid }}\right)$ definido en EPANET, y la duración de éste, en un principio limitado al máximo tiempo de riego diario ( $T_{\text {Rieg }}$ ) que es de $3 \mathrm{~h}$. Si se decide que todas las tomas de un mismo hidrante funcionan a la vez, el número de variables será dos veces el número de hidrantes $\left(N_{H}\right)$. Si $T_{\text {JRieg }}=24 \mathrm{~h}$ y $\mathrm{T}_{\text {Hid }}=900 \mathrm{~s}=15 \mathrm{~min}$, las primeras $\mathrm{N}_{\mathrm{H}}$ variables tomarán valores entre 0 y $95(24 \times 4)$. Las siguientes $N_{H}$ variables tomarán valores entre 0 y $T_{\text {Rieg, es }}$ decir, entre 0 y $12(3 \times 4)$.

\subsection{Metodología asumiendo que la presión en cabecera es constante (Met2)}

Asumiendo que la presión en cabecera es constante, o que ésta no disminuirá por debajo de un valor de referencia, se ha propuesto una nueva metodología que utiliza el modelo hidráulico de la red junto con NSGA-II, reformulado ahora para cada instante a medida que transcurre la simulación.

La hipótesis en que se basa es que cuantas más tomas funcionen, mayor será la velocidad en las tuberías y antes se alcanzarán los puntos de consumo. Del mismo modo que en el caso anterior, se asume que todas o parte de las tomas de un mismo hidrante funcionan a la vez. El volumen mínimo a inyectar será el de las tuberías de la red, $607 \mathrm{~m}^{3}$ para el caso de estudio.

Una diferencia fundamental entre este método y el anterior, es que ahora el escenario de apertura y cierre de las válvulas se va determinando a medida que transcurre el tiempo, de modo que en todo el proceso se realiza una única simulación para toda la jornada de riego. En el método anterior, en cambio, cada solución del algoritmo genético era un escenario completo para toda la jornada de riego, para el cual se evaluaban las distintas funciones objetivo a fin de compararlas con las de otros escenarios.

La metodología consta de las siguientes fases:

1. Encontrar el máximo número de tomas que pueden funcionar sin que exista DPT. Para ello se construye un algoritmo genético en el que el número de variables es $\mathrm{N}_{\mathrm{H}}$. Estas se han codificado como variables enteras y el rango de valores que pueden tomar es de 0 o 1. El valor 0 significa que el hidrante está abierto y el valor 1 que está cerrado.

2. Una vez se ha seleccionado la solución para la cual se maximiza $\mathrm{V}_{\text {Tot }} \mathrm{y}$ se minimiza $\mathrm{DPH}$, se determina el tiempo de llegada $\left(T_{H}\right)$ de la sustancia inyectada a las primeras tomas en funcionamiento.

3. Para determinar las nuevas tomas que abren se utiliza de nuevo cada vez el algoritmo genético a fin de minimizar el DPT, el cual se reinicia incorporando a la población inicial la solución de la etapa anterior, para acelerar el proceso de encontrar la mejor solución.

Para calcular $T_{H}$ en los siguientes hidrantes a los que llegará la sustancia inyectada, se reinicia desde el instante inicial el escenario en EPANET con la nueva configuración de tomas cerradas y abiertas. A medida que transcurre el tiempo, las tomas a las que va llegando la sustancia inyectada se cierran y a las que todavía no les ha llegado, se mantienen abiertas. Conforme se van cerrando algunas tomas, se abren otras de entre las restantes determinadas por el algoritmo genético.

4. El proceso se repite hasta que la sustancia llega a todas las tomas en funcionamiento. 


\subsection{Escenarios propuestos}

Para el análisis de los escenarios propuestos primero se ha evaluado el funcionamiento de la red de riego en una de las jornadas representativas de los periodos de mayor demanda, como es la primera semana de agosto. Para ello se ha calculado $V_{\text {Tot, }}$ DPT, el tiempo de llegada de la sustancia media por hidrante $\left(T_{H}\right)$, el tiempo de llegada mínimo ( $T_{H}$ Min $)$, el tiempo de llegada máximo $\left(T_{H}\right.$ Max $)$ y el número de tomas ( $N_{T_{o}} \_$Pmin $)$que funcionan por debajo de la presión mínima requerida $\left(P_{\text {Min }}\right)$, que se ha establecido en $20 \mathrm{~m}$.

Para Met1, los escenarios se configuran variando $T_{J R i e g}$ (Escenarios 2-8). $T_{\text {Rieg }}$ se ha establecido en $3 \mathrm{~h}$. No obstante, para compararla con la otra metodología propuesta, se han tomado aquellas soluciones donde se maximiza $\mathrm{N}_{\mathrm{Hs}}$. La curva de modulación de presiones en cabecera utilizada ha sido la que se muestra en la Figura 2. La población inicial seleccionada ha sido de 100 cromosomas. El número total de evaluaciones ha sido de 100000, una vez observada que la solución mejoraba levemente incrementando este valor. $T_{\text {Hid }}$ se ha establecido en $300 \mathrm{~s}$, el mismo valor para el intervalo de las curvas de modulación y el intervalo de calidad $\left(T_{Q}\right)$ en $12 \mathrm{~s}$. Se recomienda que al menos $T_{Q}$ sea la quinta parte de $T_{\text {Hid }}$ (Rossman,2000). No obstante, dadas las elevadas velocidades que se alcanzan en las tuberías, se ha establecido un $T_{Q}$ inferior al mínimo recomendado. La elección de un $T_{\text {Hid }}$ pequeño mejora el resultado para minimizar el volumen inyectado al del remplazo de las tuberías, puesto que se establece con más precisión cuando iniciar y parar el funcionamiento de las tomas de los hidrantes, si bien aumenta el número de posibles soluciones de las variables. El número posible de soluciones combinadas para un hidrante dado, considerando la hora de apertura y el tiempo que puede permanecer abierto es:

$$
N=\frac{T_{\text {Rieg }}}{T_{\text {Hid }}} \cdot \frac{T_{\text {JRieg }}}{T_{\text {Hid }}}
$$

Para Met2 (escenarios 9-17) se ha modificado el número de evaluaciones $\left(\mathrm{N}_{\mathrm{Ev}}\right)$. La presión fija establecida en el cabezal de la red ha sido la media horaria diaria, $31 \mathrm{~m}$. Se ha modificado el número de tomas de cada hidrante que funcionan a la vez ajustándose el caudal lo más aproximado al $75,50,25$ y $15 \%$ del caudal total del hidrante $(\mathrm{Q} \%)$.Se ha establecido un $T_{\text {Hid }}=60 \mathrm{~s}$ y un $T_{Q}=12 \mathrm{~s}$. Para todos los escenarios, la población inicial es de 100 cromosomas.

En ambas metodologías se ha asumido que las tomas de un mismo hidrante que funcionan, lo hacen a la vez. La probabilidad de mutación se ha establecido en un $10 \%$, el método de cruzamiento seleccionado ha sido el "SinglePointCrossover" y el método de selección el "BinaryTourment2" (Durillo y Nebro, 2011).

La inyección de la sustancia en el cabezal de la red se ha modelado como una Reinyección a Punto Fijo a la que se le ha fijado un determinado valor de la concentración.

\section{4- Análisis de los resultados}

\subsection{Análisis del funcionamiento de la red en una jornada de riego.}

La Figura 3 muestra la evolución del caudal inyectado y la presión disponible en cabecera para una jornada de riego donde operan 556 tomas distribuidas en los 88 hidrantes con un tiempo medio de funcionamiento de $3.1 \mathrm{~h}$. El $\mathrm{V}_{\text {Tot }}$ aportado es de $7803 \mathrm{~m}^{3}$, y los tiempos observados son $\mathrm{T}_{\text {Lleg } \mathrm{t} M}=3.15 \mathrm{~h}$, $\mathrm{T}_{\text {Lleg } \mathrm{t} \text { Min }}=0.33 \mathrm{~h}$ y $\mathrm{T}_{\text {Lleg } \mathrm{t} \text { Max }}=11.76 \mathrm{~h}$. Dos de los hidrantes de un ramal extremo no recibe ninguna cantidad de sustancia en las $24 \mathrm{~h}$. Un total de 317 tomas operan por debajo de $\mathrm{P}_{\text {Min. }}$. 


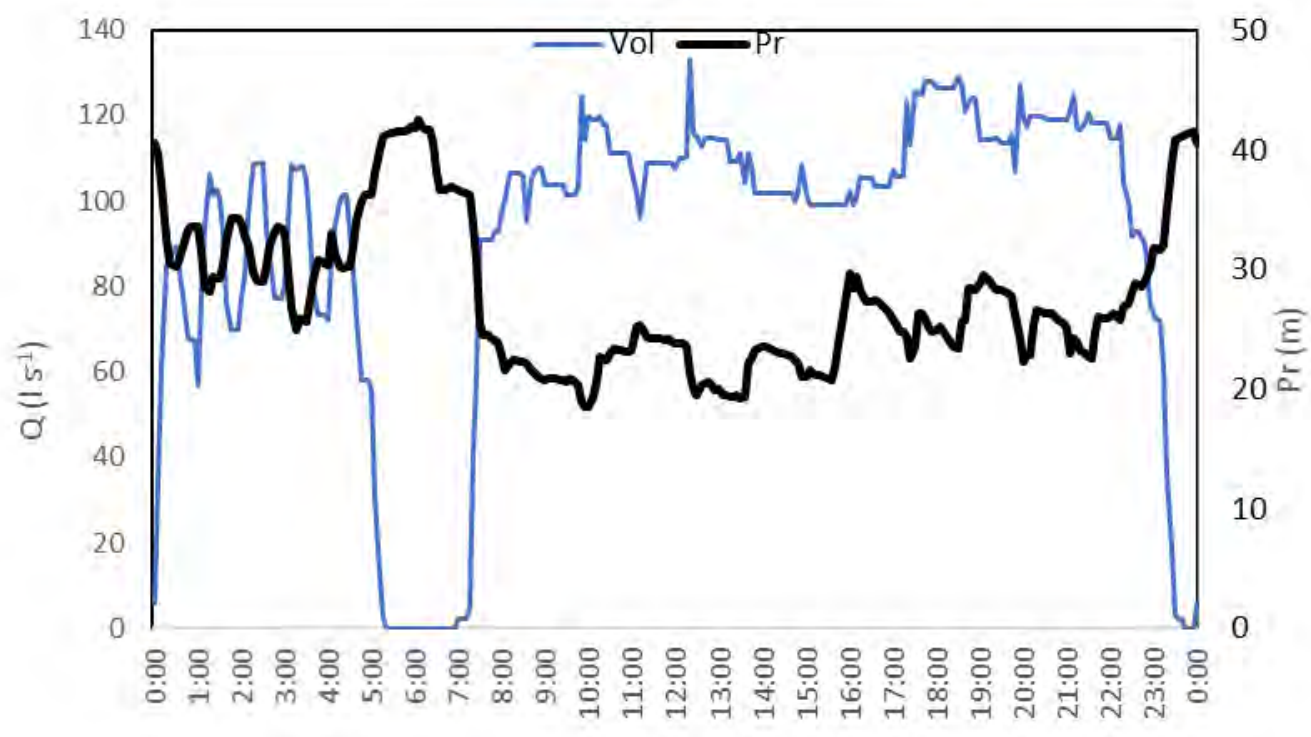

Figura 3 Caudal inyectado $\left(\mathrm{Q}, \mathrm{I} \mathrm{s}^{-1}\right)$ y presiones $(\operatorname{Pr}, \mathrm{m})$ para una jornada de riego de máxima necesidades

\subsection{Análisis de los escenarios de optimización}

La Tabla 1 muestra el resumen de los escenarios (ESC) simulados con Met1. ESC1 se corresponde con el descrito en el apartado anterior.

Tabla 1 Escenarios óptimos para diferentes valores del $\mathrm{T}_{\mathrm{JRieg}}$ en que pueden operar las tomas. Para cada uno se muestra el volumen inyectado en la red $V_{\text {Tot }}\left(\mathrm{m}^{3}\right)$, el tiempo de llegada medio de la sustancia a los hidrantes de la red $\left(T_{H M}\right)$, el tiempo mínimo ( $T_{H}$ Min), el tiempo máximo ( $T_{H}$ Max), el déficit de presión en hidrante (DPH), la duración de la jornada de riego ( $T_{J R i e g}$ y el número de hidrantes a los que le llega la sustancia $\left(\mathrm{N}_{H S}\right)$ en $\mathrm{T}_{\text {JRieg }}$.

\begin{tabular}{|c|c|c|c|c|c|c|c|}
\hline ESC & $T_{\text {JRieg }}$ & $\mathrm{V}_{\text {Tot }}\left(\mathrm{m}^{3}\right)$ & $\mathrm{T}_{H \mathrm{M}}(\mathrm{h})$ & $\mathrm{T}_{\text {M Min }}(\mathrm{h})$ & $\mathrm{T}_{H \text { Max }}(\mathrm{h})$ & $\mathrm{DPH}(\mathrm{m})$ & $\mathrm{N}_{\text {HS }}$ \\
\hline 1 & 24 & 7803 & 3.15 & 0.5 & 11.76 & 2.45 & 86 \\
\hline 2 & 24 & 1488 & 9.06 & 0.67 & 21.1 & 0.1 & 87 \\
\hline 3 & 12 & 1489 & 4.14 & 0.67 & 8.75 & 0.13 & 87 \\
\hline 4 & 10 & 1302 & 3.61 & 0.17 & 7.7 & 0 & 87 \\
\hline 5 & 8 & 1378 & 3.16 & 0.42 & 6.25 & 0.03 & 87 \\
\hline 6 & 7 & 1353 & 2.73 & 0.41 & 5.2 & 0 & 87 \\
\hline 7 & 6 & 1317 & 2.14 & 0.25 & 6 & 0.08 & 87 \\
\hline
\end{tabular}

Se observa que al aumentar $T_{\text {JRieg }}$, los tiempos de llegada aumentan y $V_{\text {Tot }}$ permanece constante, excepto para ESC 1 y 2 en los que aumenta. EL DPH muestra poca variabilidad para todos los escenarios. La Figura 4 presenta los frentes de Pareto para cada uno de los escenarios.

El máximo exceso de volumen inyectado respecto al volumen de reemplazo, debido a que las tomas se cierran después de la llegada del fertilizante porque $T_{\text {Hid }}$ no es lo suficientemente pequeño, es de $213 \mathrm{~m}^{3}$. Se obtiene al multiplicar $\mathrm{T}_{\text {Hid }}(300 \mathrm{~s})$ por la demanda base de todos los hidrantes. El resto de la diferencia entre $V_{\text {Tot }}$ y el volumen de las tuberías $\left(607 \mathrm{~m}^{3}\right)$ se debe a que el algoritmo no converge lo suficiente debido a la gran cantidad de posibles soluciones de cada variable (10368). Para reducir el número de evaluaciones, se podría acotar el rango de posibles soluciones para ciertas variables, como por ejemplo el funcionamiento de los hidrantes localizados en ramales extremos, donde el tiempo de 
funcionamiento se restringiría justo el tiempo que tarda en llegar la sustancia desde el hidrante aguas arriba.
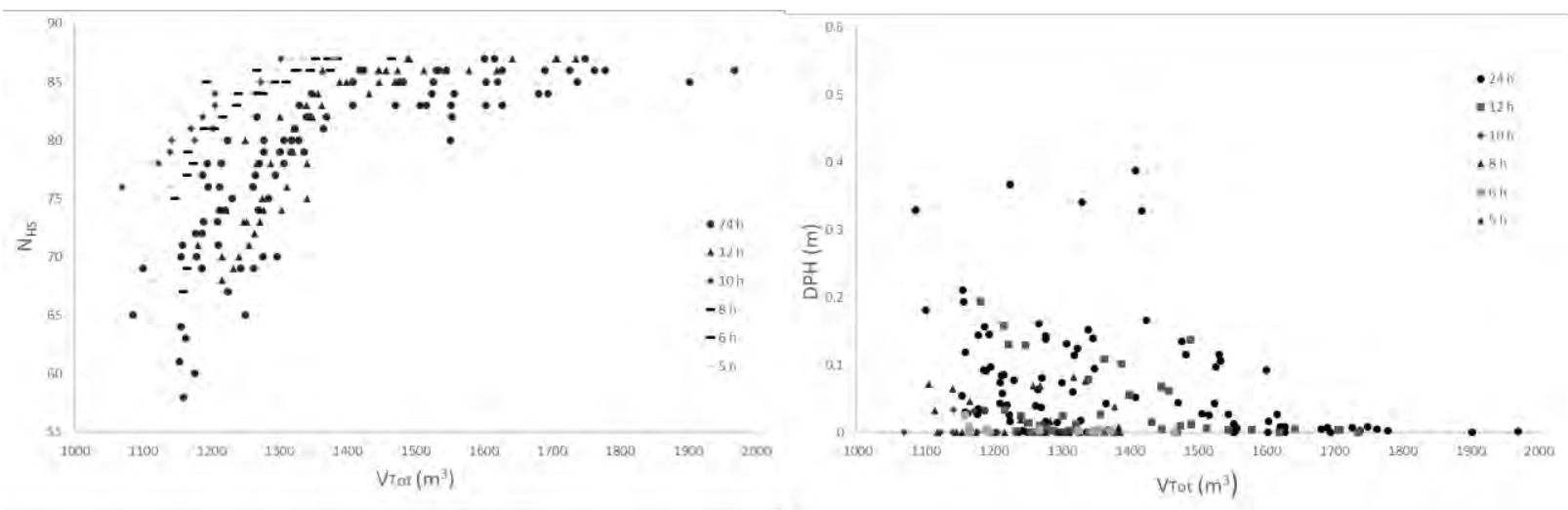

Figura 4 Volumen consumido $\left(\mathrm{Vol}, \mathrm{m}^{3}\right)$, número de hidrantes a los que les llega la sustancia ( $N_{\text {Hid_S }}$ ) y déficit de presión en hidrante para los distintas soluciones de los escenarios de

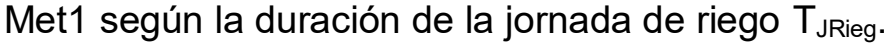

La ¡Error! No se encuentra el origen de la referencia. resume los escenarios para Met2. Para todos los escenarios propuestos la sustancia alcanza la totalidad de los hidrantes, $\mathrm{N}_{\mathrm{Hs}}=88$. El volumen inyectado para todos los escenarios coincide con el volumen total de las tuberías de la red, $607 \mathrm{~m}^{3}$.

Tabla 2 Escenarios óptimos en función del número de evaluaciones $\left(\mathrm{N}_{\mathrm{EV}}\right)$ y del caudal de las tomas. Por cada uno se muestra el tiempo de llegada medio de la sustancia a los hidrantes de la red $\left(T_{H M}\right)$, el tiempo mínimo ( $\left.T_{H \text { Min }}\right)$, el tiempo máximo ( $T_{H \text { Max }}$ ), el déficit de presión en hidrante (DPH) y el tiempo mínimo de riego que permanece abierto un hidrante.

\begin{tabular}{|c|c|c|c|c|c|c|c|}
\hline ESC & $\mathrm{N}_{\mathrm{EV}}$ & $\mathrm{Q}(\%)$ & $\mathrm{TH}_{\mathrm{M}}(\mathrm{h})$ & $\mathrm{TH}_{H}$ Min (h) & $\mathrm{TH}_{\mathrm{Max}(\mathrm{h})}$ & $\mathrm{DPH}$ & $\mathrm{T}_{\text {Rieg Min(h) }}$ \\
\hline 8 & 500 & 100 & 0.28 & 0.016 & 2.48 & 1 & 0.033 \\
\hline 9 & 1000 & 100 & 0.28 & 0.016 & 2.48 & 0.79 & 0.0166 \\
\hline 10 & 5000 & 100 & 0.27 & 0.016 & 2.46 & 0.73 & 0.033 \\
\hline 11 & 10000 & 100 & 0.27 & 0.016 & 2.46 & 0.89 & 0.033 \\
\hline 12 & 20000 & 100 & 0.27 & 0.016 & 2.46 & 0.64 & 0.033 \\
\hline 13 & 50000 & 100 & 0.27 & 0.016 & 2.46 & 0.32 & 0.033 \\
\hline 14 & 10000 & 75 & 0.36 & 0.016 & 3.25 & 0.05 & 0.033 \\
\hline 15 & 10000 & 50 & 0.52 & 0.016 & 4.92 & 0 & 0.066 \\
\hline 16 & 10000 & 25 & 0.52 & 0.016 & 9.83 & 0 & 0.083 \\
\hline 17 & 10000 & 15 & 1.72 & 0.016 & 16.4 & 0 & 0.083 \\
\hline
\end{tabular}

Cuando $Q_{\%}=100$, a medida que $N_{\mathrm{Ev}}$ aumenta $\mathrm{DPH}$ disminuye, mientras que $T_{\mathrm{H}}$ м $\mathrm{T}_{\mathrm{H}}$ Max permanecen casi inalterables. Además el tiempo que cuesta limpiar la red se reduce en $0.02 \mathrm{~h}$ comparando el escenario 8 frente al 13 (de 2.48 h 2.46 h). Esto es debido a la propia naturaleza del algoritmo genético, que al realizar más evaluaciones encuentra un mayor número de hidrantes que pueden funcionar simultáneamente. DPH se debe a un ramal de 200 $\mathrm{m}$ donde se producen pérdidas de $40 \mathrm{~m} \mathrm{~km}^{-1}$ cuando el hidrante opera a $Q_{\%}=100$.

El tiempo de limpieza de la red se completa en $2.46 \mathrm{~h}$, si bien $\mathrm{T}_{\mathrm{H} \text { M }}$ es de $0.27 \mathrm{~h}$. La presencia de ramales extremos con bajo caudal produce un aumento de la duración del proceso de limpieza. La Figura 5 muestra la evolución de los caudales en cabecera de red para ESC7, ESC11 y ESC14-17. Como se observa, Met2 concentra el funcionamiento de las tomas de los hidrantes al principio y luego va disminuyendo conforme la sustancia llega a los hidrantes y las tomas de estos cierran. 
Esto produce velocidades elevadas justo a la salida del cabezal de $5.6 \mathrm{~m} \mathrm{~s}^{-1}$, lo que puede provocar variaciones excesivas de presión al cambiar el régimen de funcionamiento de la red, sobre todo al principio de la maniobra de limpieza. Además, implica abrir un número de tomas en un tiempo muy pequeño, 1 minuto, maniobra no recomendada en la apertura de las válvulas de tomas por la corta duración. Algunos fabricantes recomiendan un funcionamiento mínimo de 5 minutos.

En los escenarios 14-17, en los que se ha reducido el número de tomas que opera a la vez en cada hidrante, se observa como los tiempos de llegada aumentan. Estos escenarios son más convenientes para llegar a todos los nudos con menores velocidades en las tuberías, pero invirtiendo más tiempo. Para ESC 17 la velocidad máxima alcanzada son $0.96 \mathrm{~m} \mathrm{~s}^{-1}$. En el caso de la aplicación de tratamientos con una sustancia reactiva con un tiempo medio de reacciónsuperior al de llegada, sería más conveniente estas soluciones para minimizar los efectos transitorios, que con $Q(\%)=100$. Además, $T_{\text {Rieg Min }}$ aumenta hasta los 5 minutos. En ESC7 la máxima velocidad en el cabezal es de1.27 m. Si se redujera la presión en cabecera, aumentarían los tiempos de llegada.

La

Figura 5 muestra la evolución del caudal inyectado a lo largo del tiempo para los

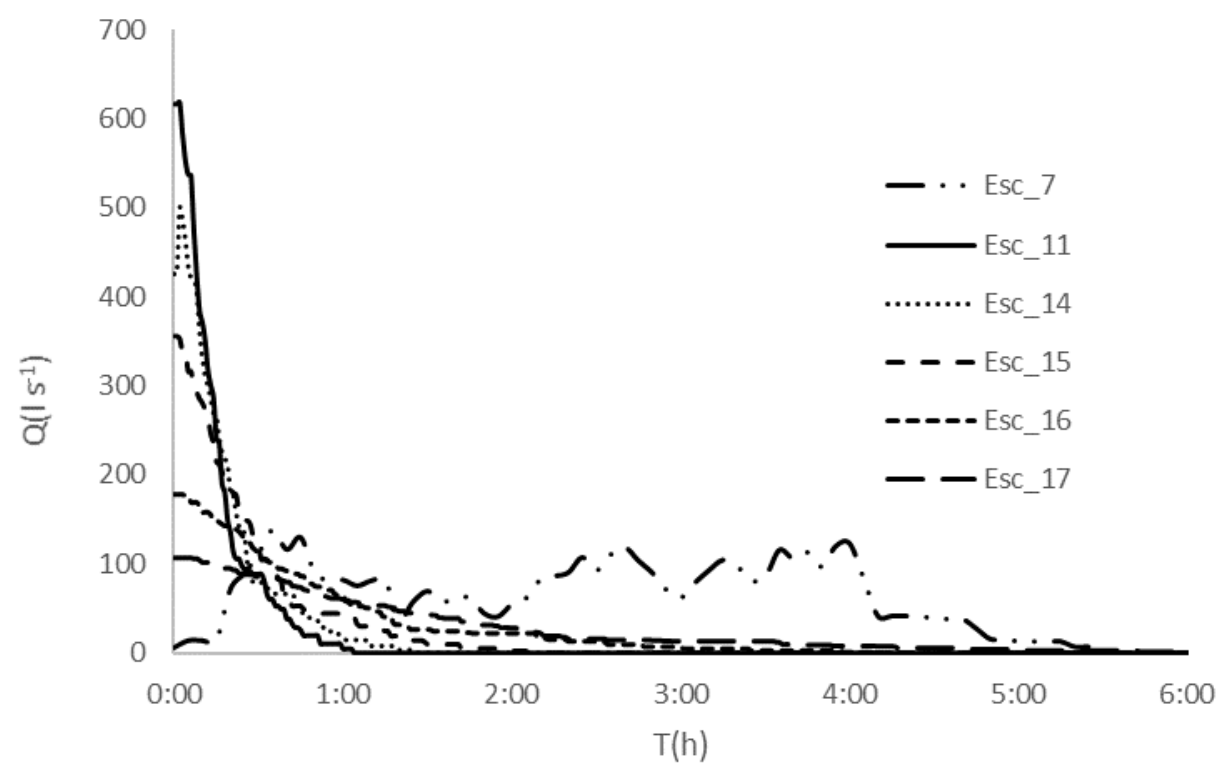

escenarios elegidos.

Figura 5 Evolución del caudal inyectado en cabecera $\left(\mathrm{Q}, \mathrm{I} \mathrm{s}^{-1}\right)$, para los Escenarios 7, 11, $14,15.16$ y 17

Met2 disminuye $\mathrm{V}_{\text {Tot }} \mathrm{y}$ los tiempos de llegada frente a Met1, si bien Met2 solo es compatible con una presión mínima garantizada en cabecera. En el caso de estudio, la red se alimenta desde una tubería general con una presión de suministro variable en el tiempo. Si el suministro se realizara con equipos de bombeo, estos podrían mantener una determinada presión de consigna en el caso de disponer de variadores de frecuencia, pero ello no sería compatible con un consumo energético mínimo. Sería necesario un estudio económico de la conveniencia de esta metodología, dependiendo de las veces en que se realicen estas operaciones de limpieza de la red, del coste de los productos y del coste energético.

Por el contrario, Met1 sí que se puede adoptar teniendo en cuenta este criterio como en Alonso Campos et al (2020) 


\section{5- Conclusiones}

En las redes de riego a presión automatizadas, ciertas operaciones requieren distribuir una sustancia a través de la red, bien para fertilizar, limpiar o desinfectar. En cualquier caso, interesa que ello se haga en el menor tiempo posible y utilizando la menor cantidad de volumen inyectado. Para ello se han desarrollado dos metodologías que utilizan el modelo matemático de la red junto con algoritmos genéticos multiobjetivo paralelos.

La primera tiene como objetivo intentar llegar al máximo número de puntos de consumo, minimizando el déficit de presión y el volumen inyectado. Además, permite que la altura en cabecera sea variable en el tiempo. La segunda tiene como objetivo llegar a todos los puntos en el menor tiempo posible respetando el déficit de presión, con la limitación de la existencia de una presión constante en cabecera.

La segunda metodología consigue mejores resultados permitiendo limpiar la totalidad de la red en un tiempo mínimo de $2.46 \mathrm{~h}$, e inyectando justo el volumen de las tuberías con un ahorro del $92.2 \%$ comparado a una jornada normal de riego (Esc 1).

\section{6- Agradecimientos}

Los autores quieren agradecer a la Acequia Real del Júcar, en especial a su técnico Alberto Hervás y a su Secretario Juan Valero de Palma, el apoyo y el interés para la realización de este trabajo.

\section{7- Bibliografía}

Alonso Campos, J. C., Jiménez-Bello, M. A., Martínez Alzamora, F. (2020). Real-time energy optimization of irrigation scheduling by parallel multi-objective genetic algorithms. Agricultural Water Management, 227. https://doi.org/10.1016/j.agwat.2019.105857

CAJAMAR 2014. Limpieza de la instalación de riego. Boletín $\mathrm{n}^{\circ}$ 114. URL: https://www.cajamar.es/pdf/bd/agroalimentario/innovacion/investigacion/documentos-yprogramas/boletin-huerto-114-1496655961.pdf

Confederación hidrográfica del Ebro 2014. Mejillón cebra; Manual de control para instalaciones afectadas. $2^{\mathrm{a}}$ ed. Zaragoza. $42 \mathrm{pp}$

http://www.chebro.es/contenido.streamFichero.do?idBinario=18109

Deb, K., Pratap, A., Agarwal, S., Meyarivan, T., 2002. A fast and elitist multiobjective genetic algorithm: NSGA-II. IEEE Trans. Evol. Comput. 6, 182-197.

https://ieeexplore.ieee.org/document/996017

Durillo, J.J., Nebro, A.J., 2011. JMetal: a Java framework for multi-objective optimization. Adv. Eng. Softw. 42, 760-771.

https://www.sciencedirect.com/science/article/abs/pii/S0965997811001219

Jimenez-Bello, M. A., Martínez, F., Bou, V., \& Bartolín, H. (2011). Analysis, assessment, and improvement of fertilizer distribution in pressure irrigation systems. Irrigation Science, 29(1) https://link.springer.com/article/10.1007/s00271-010-0215-7

Rossman, L. A. (2000). Epanet 2, users manual. Cincinnati, USA:Water Supply and Water Resources Division. National Risk. Management Research Laboratory.

https://www.epa.gov/water-research/epanet 